\title{
Experiencia en Costa Rica con la detección de mutaciones en el gen KRas en pacientes oncológicos: I reporte
}

Madrigal Sánchez $\mathrm{JJ}^{1}$, Salazar Salas $\mathrm{L}^{1}$, Calvo Flores $\mathrm{L}^{1}$, Porras Peñaranda $\mathrm{J}^{1-2}$

Las proteínas RAS son pequeñas GTPasas que alternan entre KRAS unido a GTP en el estado activo y posee una actividad enzimática intrínseca que escinde el fosfato terminal del nucleótido convirtiéndole a GDP. Tras la conversión de GTP a GDP, KRAS se torna inactivo. Las proteínas RAS son mediadores centrales downstream de la señalización del receptor del factor de crecimiento epidérmico (EGFR) y por lo tanto son críticos para la proliferación celular, la supervivencia y la diferenciación. RAS puede activar varios efectores downstream, incluyendo la vía PI3K-AKT-mTOR, que está implicada en la supervivencia celular, y de la vía RAS-RAF-MEK-ERK, que está implicado en la proliferación celular (ver Figura 1).

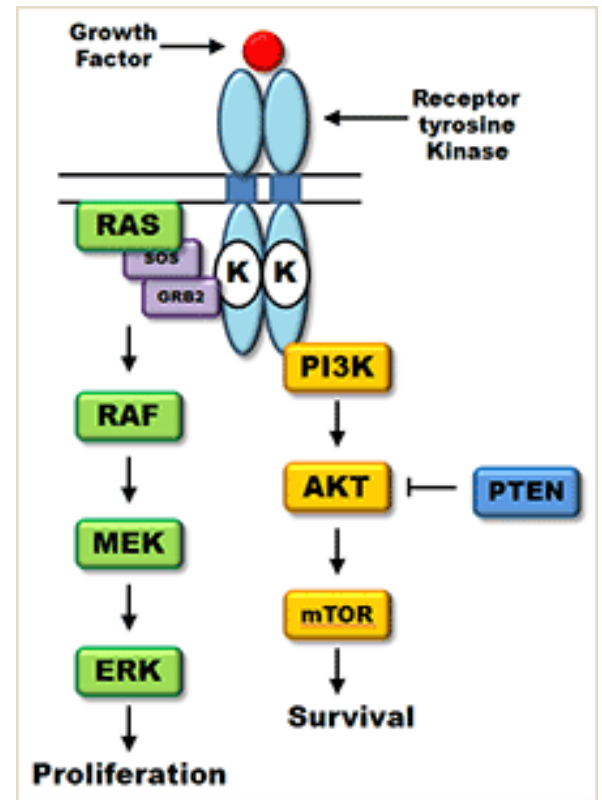

Figura 1. Esquema de las vías de MAPK y PI3K. Factor de crecimiento de unión a los receptores de los resultados de la tirosina quinasa en la activación de la vía de señalización MAPK (RAS-RAF-MEK-ERK) y la vía PI3K (PI3K-AKT-mTOR). La letra "K" dentro del esquema denota el dominio tirosina quinasa.

Los genes RAS han sido implicados en la patogénesis de varios tipos de cáncer. La aparición de mutaciones en el gen RAS da como resultado la activación constitutiva de la RAS-GTPasa, incluso en ausencia del factor de crecimiento epidérmico. El resultado de dicha mutación es una señal de proliferación permanente dentro de la célula.

Los genes específicos RAS se encuentran recurrentemente mutados en diferentes tumores malignos. Las mutaciones KRAS son especialmente frecuentes en el cáncer de colon, pulmón y páncreas o en los procesos metastásicos de los mismos $(1,2)$. 
Hoy día esta descrito que cerca del $36-40 \%$ de los pacientes con cáncer colorrectal tienen mutaciones del gen KRAS asociados a tumores $(3,4,5,6)$. La mayoría de las mutaciones se producen en los codones 12 y 13 del gen KRAS.

Múltiples estudios han demostrado ahora que los pacientes con tumores que albergan mutaciones en KRAS poseen pocas probabilidades de beneficiarse de la terapia con anticuerpos monoclonales anti-EGFR, ya sea como monoterapia o en combinación con quimioterapia $(7,8,9)$. Además, en los ensayos de quimioterapia basada en oxaliplatino, los pacientes con tumores KRAS mutados parecían empeorar su condición cuando son tratados con terapia de anticuerpos anti-EGFR en combinación con una quimioterapia basada en oxaliplatino en comparación con los pacientes tratados con un tratamiento con oxaliplatino únicamente. El tratamiento con cetuximab, un anticuerpo monoclonal dirigido contra el EGFR, mejora la supervivencia global y libre de progresión y conserva la calidad de vida en pacientes con cáncer colorrectal que no ha respondido a la quimioterapia. El estado de la mutación del gen K-ras en el tumor puede afectar a la respuesta a cetuximab y tienen-tratamiento valor pronóstico independiente $(10,11,12)$.

Para el caso de Costa Rica no existe ninguna estadística sobre la prevalencia de mutaciones en el gen KRAS, por eso resulta muy importante la determinación de la misma para poder correlacionar con la importancia clínica, en el sentido de que dependiendo del estado mutacional del paciente con cáncer de colon, así debería ser la terapia con la que vaya a ser tratado. Dicho procedimiento tiene sus aristas y por esa razón lo primero que debe de hacerse es estandarizar la prueba para las condiciones de los laboratorios del país.

Precisamente ante este vacío de conocimiento es que se decide estandarizar la prueba molecular para la determinación de la mutación de KRAS en los pacientes con cáncer de colon metastásico.

\section{Metodología}

Se obtiene una biopsia del tumor del paciente, dicha biopsia es analizada por el patólogo quién determina la zona tumoral y si se tiene al menos un $50 \%$ de esta en la totalidad de la lámina (muestras con menos del $50 \%$ de tumor no son adecuadas para el estudio).

Posteriormente al análisis patológico y cuando se determina que la biopsia es apta para el estudio se procede a realizar 5 laminas de la misma biopsia, dichas laminas son de 5 um de grosor, desparafinadas y sin tinción.

Las laminas son enviadas al CIHATA para el estudio molecular que inicia con la microdisección de únicamente la zona tumoral, dicha microdisección demaracará la zona de la cual se hara la extracción del ADN. 
Una vez extraído el material genético se procede con la cuantificación del mismo, para determinar la concentración de ADN y de materiales contaminantes (restos proteicos principalmente). Luego de cuantificar el material y determinar que es la concentración adecuada se hace la amplificación (termociclador AB-9700) de la región de KRAS.

Posteriormente se hace una purificación del amplicón, y por último la reacción de secuenciación, para posteriormente purificar e introducir al analizador genético (ABI310).

Posteriormente las secuencias de los codones 12 y 13 son analizadas para buscar mutaciones, en caso de presencia de mutaciones se compara con la base de datos existente para KRAS (13) esto con la intención de determinar si la misma ya esta descrita o si es una mutación aun no descrita. Por último se elabora el informe indicando el estado mutacional del gen KRAS.

\section{Resultados}

Se analizaron 73 muestras, de las cuales 44 resultaron normales (no mutadas) y 5 mostraron alguna mutación en los codones 12 o 13; las 23 restantes resultaron ser muestras inadecuadas.

En el caso de las muestras inadecuadas se descartaron para el análisis y la gran mayoría fueron descartadas porque no se llegó a la concentración mínima requerida para llevar a cabo el estudio molecular. Otra de las razones por las cuales no fueron tomadas en cuenta fue porque no llegaron al $50 \%$ mínimo de parte tumoral de la totalidad de la biopsia, y la última de las razones fue la elevada concentración de contaminantes (restos proteicos) provenientes de la biopsia.

En el caso de las muestras mutadas se obtuvieron las siguientes mutaciones:

- Mutación puntual en el codón 13 del gen KRAS producido por un cambio de un nucleótido guanina (G) a una adenina (A) correspondiendo a un cambio de aminoácido de Glicina por Aspartato.

- mutación puntual en el codón 12 del gen KRAS producido por un cambio de un nucleótido glicina (G) a una adenina (A) correspondiendo a un cambio de aminoácido de Glicina por Aspartato.

- mutación puntual en el codón 13 del gen KRAS producido por un cambio de un nucleótido guanina $(\mathbf{G})$ a una citosina $(C)$ correspondiendo a una mutación GGC $\rightarrow$ CGC afectando al aminoácido de Glicina con cambio a Arginina.

- mutación puntual en el codón 12 del gen KRAS producido por un cambio de un nucleótido guanina (G) a una timina(T) correspondiendo a una mutación GGT $\rightarrow$ GTT afectando al aminoácido de Glicina con cambio a Valina. 
- mutación puntual en el codón 13 del gen KRAS producido por un cambio de un nucleótido glicina $(\mathbf{G})$ a una adenina $(\mathbf{A})$ correspondiendo a un cambio de aminoácido de Glicina por Aspartato.

Todas las mutaciones antes señaladas estaban previamente descritas y documentadas, por lo que no se registró ninguna mutación no descrita previamente.

\section{Conclusiones}

El estudio molecular del gen KRAS es de suma importancia para determinar le mejor terapia para pacientes con cáncer colorrectal, aunque es importante tomar en cuenta que no todo paciente con cáncer de colon es sujeto de estudio para dicho análisis, ya que la mutación en KRAS es de tipo somática, y además es de las más tardías en aparecer, por lo que los sujetos con cáncer colorrectal metastásico son los más indicados para dicho análisis. Sumado a lo anterior se debe valorar el componente costo-beneficio de la prueba, ya que hacer el estudio para pacientes que (por lo temprano del proceso tumoral) no han expresado la mutación en KRAS nos daría una falsa impresión del estado de dicha región génica (no mutado), y en algún momento podría expresarla y con esto afectar el curso de la terapia monoclonal que está indicada para estos pacientes.

En Costa Rica no existen antecedentes sobre la presencia de estas mutaciones en la población con cáncer colorrectal (en ninguna de las etapas del proceso tumoral), esto aunado a la importancia del estudio para la determinación de la terapia a recibir del paciente hace que se vuelva de suma importancia instaurar esta prueba para la población costarricense, se debe sumar el hecho de que el sistema de seguridad social del país ya cuenta con el anticuerpo monoclonal para darle a estos pacientes.

Los análisis multi-disciplinarios tienen un mayor valor para la calidad de vida del paciente, y este ejemplo del análisis de KRAS es un claro ejemplo, en el que el oncólogo determina el tipo de cáncer en cuestión, posteriormente un cirujano extrae la biopsia de la región afectada, dicha biopsia es analizada por patología para determinar exactamente la región tumoral, para de esa región específicamente hacer el estudio molecular. Este tipo de intervención es personalizada, pues dependerá del perfil obtenido al final el cómo cada paciente sea tratado, según el background genético del mismo.

Este es un primer informe para el análisis del estado mutacional del gen KRAS, los retos siguen estando presentes: aumentar la cantidad de muestras a analizar para poder determinar prevalencias, establecer un plan nacional para determinar el protocolo como serán tratados los pacientes con cáncer colonorectal. 


\section{Bibliografía}

1. Karnoub AE, Weinberg RA (2008). Ras oncogenes: split personalities. Nat Rev Mol Cell Biol. Jul; 9(7):517-31.

2. Schubbert S, Shannon K, Bollag G (2007). Hyperactive Ras in developmental disorders and cancer. Nat Rev Cancer. Apr; 7(4):295-308.

3. Amado RG, Wolf M, Peeters M, Van Cutsem E, et al (2008). Wild-type KRAS is required for panitumumab efficacy in patients with metastatic colorectal cancer. J Clin Oncol. Apr 1; 26(10):1626-34.

4. http://cancer.sanger.ac.uk/cosmic/gene/overview?In=KRAS, 07/10/2014

5. http://meetinglibrary.asco.org/content/60973-101, 07/10/2014

6. Neumann J, Zeindl-Eberhart E, Kirchner T, Jung A. (2009). Frequency and type of KRAS mutations in routine diagnostic analysis of metastatic colorectal cancer. Pathol Res Pract. ; 205(12):85862.

7. Bokemeyer C, Bondarenko I, Makhson A, et al (2009). Fluorouracil, leucovorin, and oxaliplatin with and without cetuximab in the first-line treatment of metastatic colorectal cancer. J Clin Oncol. Feb $10 ; 27(5): 663-71$.

8. Amado RG, Wolf $M$, Peeters $M$, et al (2008). Wild-type KRAS is required for panitumumab efficacy in patients with metastatic colorectal cancer. J Clin Oncol. Apr 1;26(10):1626-34.

9. Bokemeyer C, Bondarenko I, Hartmann JT, et al (2011). Efficacy according to biomarker status of cetuximab plus FOLFOX-4 as first-line treatment for metastatic colorectal cancer: the OPUS study. Ann Oncol. Jul; 22(7):1535-46.

10. Douillard JY, Siena S, Cassidy J, et al (2010). Randomized, phase III trial of panitumumab with infusional fluorouracil, leucovorin, and oxaliplatin (FOLFOX4) versus FOLFOX4 alone as first-line treatment in patients with previously untreated metastatic colorectal cancer: the PRIME study. J Clin Oncol. Nov 1;28(31):4697-705.

11. Lièvre A, Bachet JB, Le Corre D, et al (2006). KRAS mutation status is predictive of response to cetuximab therapy in colorectal cancer. Cancer Res. Apr 15; 66(8):3992-5.

12. Peeters M, Price TJ, Cervantes A, et al (2010) Randomized phase III study of panitumumab with fluorouracil, leucovorin, and irinotecan (FOLFIRI) compared with FOLFIRI alone as second-line treatment in patients with metastatic colorectal cancer.J Clin Oncol. Nov 1; 28(31):4706-13.

13. http://www.mycancergenome.org, 07/10/2014 
